\title{
PERIODICITY IN A RATIO-DEPENDENT PREDATOR-PREY SYSTEM WITH STAGE STRUCTURE FOR PREDATOR
}

\author{
FENGDE CHEN
}

Received 27 August 2003 and in revised form 11 September 2004

With the help of a continuation theorem based on Gaines and Mawhin's coincidence degree, easily verifiable criteria are established for the global existence of positive periodic solutions of a delayed ratio-dependent predator-prey system with stage structure for predator. The approach involves some new technique of priori estimate. For the system without delay, by constructing a suitable Lyapunov function, some sufficient conditions which guarantee the existence of a unique global attractive positive periodic solution are obtained. Those results have further applications in population dynamics.

\section{Introduction}

Recently, some biologists have argued that the ratio-dependent predator-prey model

$$
\begin{aligned}
& \frac{d x}{d t}=x(a-b x)-\frac{c x y}{m y+x}, \\
& \frac{d y}{d t}=y\left(-d+\frac{f x}{m y+x}\right)
\end{aligned}
$$

is more appropriate than the Gause type model for modelling predator-prey interactions when predation involves searching processes. This is strongly supported by numerous laboratory experiments and observations $[1,2,3,4,13,18]$. Many authors $[1,5,8,17]$ have observed that system (1.1) exhibits much richer, more complicated, and more reasonable or acceptable dynamics. Beretta and Kuang [5] introduced a single discrete-time delay into the predator equation in the above model, that is,

$$
\begin{gathered}
\frac{d x}{d t}=x(a-b x)-\frac{c x y}{m y+x} \\
\frac{d y}{d t}=y\left(-d+\frac{f x(t-\tau)}{m y(t-\tau)+x(t-\tau)}\right)
\end{gathered}
$$

and carried out systematic works on the global qualitative analysis of (1.2). 
Many excellent works have been done for the ratio-dependent predator-prey system, with or without time delay, in the sense that most results are constant environmentrelated (see $[24,25,28,27,29]$ ). Recently, more and more scholars pay attention to the nonautonomous cases of ratio-dependent predator-prey system (cf. [11, 15, 19, 20, 21]). For example, Fan et al. [15] studied the nonautonomous cases of system (1.1). For the general nonautonomous case, they gave a thoroughly analysis on the positive invariance, permanence, nonpersistence, and global stability of the system. For the periodic case or almost periodic case, by using the coincidence degree theory and constructing a suitable Lyapunov function, they obtained sufficient conditions which guarantee the existence, uniqueness, and stability of periodic solution and almost periodic solution of the system. Also, in [14], they further considered system (1.2) with time-varying delays, by using the coincidence degree theory, they obtained sufficient conditions which guarantee the existence of positive periodic solution. Their result shows that delay has no influence on the existence of positive periodic solution of the system.

Though many excellent works have been done for the ratio-dependent predator-prey system (cf. $[11,14,15,19,20,21,24,25,27,28,29]$ ), such models are not well studied in the sense that most scholars did not consider the influence of stage structure on species, that is, all the above-mentioned works assumed that each individual predator admits the same ability to attack prey. Already, there were many scholars considering the stage-structure ecosystem. In [23], a predator-prey system with stage structure was established under the assumptions that the predator is divided into two groups, one immature and the other mature, and that the mature predator attacks the prey and admits reproductive ability, while the immature predator does not attack the prey and does not have reproductive ability. It is found that an orbitally asymptotically stable periodic orbit exists in the model. When time delay due to gestation of the predator and time delay from crowding effect of the prey are incorporated, they established the condition for the permanence of populations and obtained sufficient conditions under which positive equilibrium of the model is globally stable. In [22], they further incorporated Holling-II-type functional response into the model and investigated extinction and permanence of the model. Stimulated by the works of $[22,23]$, in [26], Xiao considered the ratio-dependent predator-prey system with stage structure, that is

$$
\begin{gathered}
\frac{d x}{d t}=x(r-a x)-\frac{b x y_{2}}{m y_{2}+x}, \\
\frac{d y_{1}}{d t}=\frac{k b x y_{2}}{m y_{2}+x}-\left(D+v_{1}\right) y_{1}, \\
\frac{d y_{2}}{d t}=D y_{1}-v_{2} y_{2} .
\end{gathered}
$$

Xiao found that system (1.3) has a more complicate dynamics behavior.

However, to the best of the authors' knowledge, to this day, still no scholar considers the nonautonomous case of system (1.3), though the nonautonomous case is more realistic. So, in the present paper, we consider a more general delayed ratio-dependent 
predator-prey model with periodic coefficients and stage structure of the predator:

$$
\begin{gathered}
\frac{d x(t)}{d t}=x(t)(r(t)-a(t) x(t))-\frac{b(t) x(t) y_{2}(t)}{m y_{2}(t)+x(t)} \\
\frac{d y_{1}(t)}{d t}=\frac{c(t) x(t-\tau(t)) y_{2}(t-\tau(t))}{m y_{2}(t-\tau(t))+x(t-\tau(t))}-\left(D(t)+v_{1}(t)\right) y_{1}(t) \\
\frac{d y_{2}(t)}{d t}=D(t) y_{1}(t)-v_{2}(t) y_{2}(t)
\end{gathered}
$$

where $x(t)$ is the density of the prey, $y_{1}(t)$ is the density of the immature predator, and $y_{2}(t)$ is the density of the mature predator. $r(t), a(t), b(t), c(t), D(t), v_{1}(t)$, and $v_{2}(t)$ are all continuously positive periodic functions with periodic $\omega . r(t)$ is the intrinsic growth rate of the prey, $v_{1}(t)$ is the death rate of the immature predator, and $v_{2}(t)$ is the death rate of the mature predator, $D(t)$ denotes the rate of the immature predator to be a mature predator, it is assumed that this rate is proportional to the density of the immature predator. $k(t)=c(t) / b(t)$ denotes the conversion rate of nutrients into the reproduction of the predator, $\tau(t)$ is a nonnegative continuous $\omega$-periodic function denotes the time delay due to gestation of the mature predator. We have assumed in (1.4) that, when the predator is absent, the prey species is governed by the well-known logistic equation, in which each individual competes with all others for common resources. We have also assumed that the functional response of a predator is of a Michaelis-Menten type. In the absence of prey species, the average growth rate of the immature predator species decreases exponentially due to the presence of $-\left[D(t)+v_{1}(t)\right]$ in the immature predator dynamics, and so the average growth rate of the mature predator species decreases exponentially due to the presence of $-v_{2}(t)$ in the mature predator dynamics.

The initial conditions for system (1.4) take the form of

$$
\begin{array}{ccc}
x(\theta) & =\phi(\theta) \geq 0, \quad y_{1}(\theta)=\psi_{1}(\theta) \geq 0, \quad y_{2}(\theta)=\psi_{2}(\theta) \geq 0, \\
\theta & \in[-\tau, 0], \quad \phi(0)>0, \quad \psi_{1}(0)>0, \quad \psi_{2}(0)>0,
\end{array}
$$

where $\tau=\max _{0 \leq t \leq \omega}\{\tau(t)\}, \Phi=\left(\phi(\theta), \psi_{1}(\theta), \psi_{2}(\theta)\right) \in C\left([-\tau, 0], R_{+0}^{3}\right)$, where $R_{+0}^{3}=\left\{\left(x_{1}\right.\right.$, $\left.\left.x_{2}, x_{3}\right): x_{i} \geq 0, i=1,2,3\right\}$. By similar arguments as those of [23, Lemma 2.3], one can prove that the solution of system (1.4) with initial conditions (1.5) exists and remains positive for all future time.

The organization of this paper is as follows. Section 2 is devoted to deriving a set of easily verifiable sufficient conditions for the global existence of positive periodic solutions of (1.4) with initial conditions (1.5). The method used here will be the coincidence degree theory developed by Gaines and Mawhin [16] and some new technique of priori estimate. This method was elegantly applied by Chen [12] on a competition model, and his success inspired us. Section 3 deals with the system under the assumption $\tau(t) \equiv 0$, by constructing a suitable Lyapunov function, sufficient conditions are obtained which guarantee the existence of a unique globally attractive positive periodic solution of the system. A brief discussion is given at the end of the paper. For more works on 
periodic solution of ecosystem, one could refer to $[6,7,8,9,10]$ and the references cited therein.

\section{Existence of positive periodic solutions}

In order to obtain the existence of positive periodic solutions of (1.4), for the reader's convenience, we will summarize in the following a few concepts and results from [16] that will be basic for this section.

Let $X, Z$ be normed vector spaces, $L:$ Dom $L \subset X \rightarrow Z$ a linear mapping, and $N: X \rightarrow Z$ a continuous mapping. The mapping $L$ will be called a Fredholm mapping of index zero if $\operatorname{dim} \operatorname{Ker} L=\operatorname{Codim} \operatorname{Im} L<+\infty$ and $\operatorname{Im} L$ is closed in $Z$. If $L$ is a Fredholm mapping of index zero, there exist continuous projectors $P: X \rightarrow X$ and $Q: Z \rightarrow Z$ such that $\operatorname{Im} P=\operatorname{Ker} L$, $\operatorname{Im} L=\operatorname{Ker} Q=\operatorname{Im}(I-Q)$. It follows that $L \mid \operatorname{Dom} L \cap \operatorname{Ker} P:(I-P) X \rightarrow \operatorname{Im} L$ is invertible. We denote the inverse of that map by $K_{P}$. If $\Omega$ is an open bounded subset of $X$, the mapping $N$ will be called $L$-compact on $\bar{\Omega}$ if $Q N(\bar{\Omega})$ is bounded and $K_{P}(I-Q) N: \bar{\Omega} \rightarrow X$ is compact. Since $\operatorname{Im} Q$ is isomorphic to $\operatorname{Ker} L$, there exists an isomorphism $J: \operatorname{Im} Q \rightarrow$ $\operatorname{Ker} L$.

In the proof of our existence theorem below, we will use the continuation theorem of Gaines and Mawhin (see [16, page 40]).

LeMma 2.1 (the continuation theorem). Let $L$ be a Fredholm mapping of index zero and let $N$ be L-compact on $\bar{\Omega}$. Suppose

(a) for each $\lambda \in(0,1)$, every solution $x$ of $L x=\lambda N x$ is such that $x \notin \partial \Omega \cap \operatorname{Dom} L$;

(b) $Q N x \neq 0$ for each $x \in \partial \Omega \cap \operatorname{Ker} L$ and

$$
\operatorname{deg}\{J Q N, \Omega \cap \operatorname{Ker} L, 0\} \neq 0 .
$$

Then the equation $L x=N x$ has at least one solution lying in $\operatorname{Dom} L \cap \bar{\Omega}$.

For convenience, we introduce the notations

$$
\bar{g}=\frac{1}{\omega} \int_{0}^{\omega} g(t) d t, \quad g^{l}=\min _{t \in R} g(t)=\min _{t \in[0, \omega]} g(t), \quad g^{u}=\max _{t \in R} g(t)=\max _{t \in[0, \omega]} g(t),
$$

where $g$ is a continuous $\omega$-periodic function.

THEOREM 2.2. If

(i) $m r^{l}>b^{u}$,

(ii) $c^{l} D^{l}>v_{2}^{u}\left(D^{u}+v_{1}^{u}\right)$,

hold, then system (1.4) with initial condition (1.5) has at least one positive w-periodic solution.

Proof. Making the change of variables

$$
x(t)=\exp \{u(t)\}, \quad y_{i}(t)=\exp \left\{n_{i}(t)\right\}, \quad i=1,2,
$$


then (1.4) can be reformulated as

$$
\begin{gathered}
\frac{d u(t)}{d t}=r(t)-a(t) \exp \{u(t)\}-\frac{b(t) \exp \left\{n_{2}(t)\right\}}{m \exp \left\{n_{2}(t)\right\}+\exp \{u(t)\}}, \\
\frac{d n_{1}(t)}{d t}=\frac{c(t) \exp \{u(t-\tau(t))\} \exp \left\{n_{2}(t-\tau(t))-n_{1}(t)\right\}}{m \exp \left\{n_{2}(t-\tau(t))\right\}+\exp \{u(t-\tau(t))\}}-\left(D(t)+v_{1}(t)\right), \\
\frac{d n_{2}(t)}{d t}=D(t) \exp \left\{n_{1}(t)-n_{2}(t)\right\}-v_{2}(t) .
\end{gathered}
$$

In order to apply Lemma 2.1 (the continuation theorem) to (2.4), we first define

$$
\begin{gathered}
X=Z=\left\{x(t)=\left(u(t), n_{1}(t), n_{2}(t)\right)^{T} \in C\left(R, R^{3}\right), x(t+\omega)=x(t)\right\}, \\
\|x\|=\max _{t \in[0, \omega]}\left|n_{1}(t)\right|+\max _{t \in[0, \omega]}\left|n_{2}(t)\right|+\max _{t \in[0, \omega]}|u(t)|
\end{gathered}
$$

for any $x \in X($ or $Z)$. Then $X$ and $Z$ are Banach spaces with the norm $\|\cdot\|$. For any $x \in X$, because of the periodicity, we can easily check that

$$
\begin{gathered}
\Delta_{1}(x, t)=r(t)-a(t) \exp \{u(t)\}-\frac{b(t) \exp \left\{n_{2}(t)\right\}}{m \exp \left\{n_{2}(t)\right\}+\exp \{u(t)\}} \in C(R, R), \\
\Delta_{2}(x, t)=\frac{c(t) \exp \{u(t-\tau(t))\} \exp \left\{n_{2}(t-\tau(t))-n_{1}(t)\right\}}{m \exp \left\{n_{2}(t-\tau(t))\right\}+\exp \{u(t-\tau(t))\}}-\left(D(t)+v_{1}(t)\right) \in C(R, R), \\
\Delta_{3}(x, t)=D(t) \exp \left\{n_{1}(t)-n_{2}(t)\right\}-v_{2}(t) \in C(R, R)
\end{gathered}
$$

are all $\omega$-periodic. Let

$$
\begin{gathered}
N x=\left(\Delta_{1}(x, t), \Delta_{2}(x, t), \Delta_{3}(x, t)\right)^{T}, \quad x \in X . \\
L x=\dot{x}=\frac{d x(t)}{d t}, \quad P x=\frac{1}{\omega} \int_{0}^{\omega} x(t) d t, \quad x \in X, \quad Q z=\frac{1}{\omega} \int_{0}^{\omega} z(t) d t, \quad z \in Z .
\end{gathered}
$$

Then it follows that

$$
\begin{gathered}
\operatorname{Ker} L=R^{3}, \quad \operatorname{Im} L=\left\{z \in Z: \int_{0}^{\omega} z(t) d t=0\right\} \text { is closed in } Z, \\
\operatorname{dimKer} L=3=\operatorname{CodimIm} L,
\end{gathered}
$$

and $P, Q$ are continuous projectors such that

$$
\operatorname{Im} P=\operatorname{Ker} L, \quad \operatorname{Ker} Q=\operatorname{Im} L=\operatorname{Im}(I-Q) .
$$

Therefore, $L$ is a Fredholm mapping of index zero. Furthermore, the inverse (to $L$ ) $K_{P}$ : $\operatorname{Im} L \rightarrow \operatorname{Ker} P \cap \operatorname{Dom} L$ exists and has the form

$$
K_{P}(z)=\int_{0}^{t} z(s) d s-\frac{1}{\omega} \int_{0}^{\omega} \int_{0}^{t} z(s) d s d t .
$$


Thus

$$
\begin{aligned}
Q N x= & \left(\frac{1}{\omega} \int_{0}^{\omega} \Delta_{1}(x, \tau) d \tau, \frac{1}{\omega} \int_{0}^{\omega} \Delta_{2}(x, \tau) d \tau, \frac{1}{\omega} \int_{0}^{\omega} \Delta_{3}(x, \tau) d \tau\right)^{T}, \\
& K_{P}(I-Q) N x=\left(\Phi_{1}(x, t), \Phi_{2}(x, t), \Phi_{3}(x, t)\right)^{T},
\end{aligned}
$$

where $\Phi_{i}(x, t)=\int_{0}^{t} \Delta_{i}(x, s) d s-1 / \omega \int_{0}^{\omega} \int_{0}^{t} \Delta_{i}(x, s) d s d t-(t / \omega-1 / 2) \int_{0}^{\omega} \Delta_{i}(x, s) d s, i=1,2,3$. Obviously, $Q N$ and $K_{P}(I-Q) N$ are continuous. It is not difficult to show that $\overline{K_{P}(I-Q) N(\bar{\Omega})}$ is compact for any open bounded $\Omega \subset X$ by using the Arzela-Ascoli theorem. Moreover, $Q N(\bar{\Omega})$ is clearly bounded. Thus, $N$ is $L$-compact on $\bar{\Omega}$ with any open bounded set $\Omega \subset X$.

Now we reach the position to search for an appropriate open bounded subset $\Omega$ for the application of the continuation theorem (Lemma 2.1). Corresponding to the operator equation $L x=\lambda N x, \lambda \in(0,1)$, we have

$$
\begin{gathered}
\frac{d u(t)}{d t}=\lambda\left[r(t)-a(t) \exp \{u(t)\}-\frac{b(t) \exp \left\{n_{2}(t)\right\}}{m \exp \left\{n_{2}(t)\right\}+\exp \{u(t)\}}\right], \\
\frac{d n_{1}(t)}{d t}=\lambda\left[\frac{c(t) \exp \{u(t-\tau(t))\} \exp \left\{n_{2}(t-\tau(t))-n_{1}(t)\right\}}{m \exp \left\{n_{2}(t-\tau(t))\right\}+\exp \{u(t-\tau(t))\}}-\left(D(t)+v_{1}(t)\right)\right], \\
\frac{d n_{2}(t)}{d t}=\lambda\left[D(t) \exp \left\{n_{1}(t)-n_{2}(t)\right\}-v_{2}(t)\right] .
\end{gathered}
$$

Assume that $x=x(t) \in X$ is a solution of (2.12) for a certain $\lambda \in(0,1)$. Since $x \in X$, there exist $t_{i} \in[0, \omega]$ such that

$$
u\left(t_{1}\right)=\max _{t \in[0, \omega]} u(t), \quad n_{1}\left(t_{2}\right)=\max _{t \in[0, \omega]} n_{1}(t), \quad n_{2}\left(t_{3}\right)=\max _{t \in[0, \omega]} n_{2}(t) .
$$

Then one has

$$
\begin{aligned}
& 0=\left.\frac{d u(t)}{d t}\right|_{t=t_{1}}=\lambda\left[r\left(t_{1}\right)-a\left(t_{1}\right) \exp \left\{u\left(t_{1}\right)\right\}-\frac{b\left(t_{1}\right) \exp \left\{n_{2}\left(t_{1}\right)\right\}}{m \exp \left\{n_{2}\left(t_{1}\right)\right\}+\exp \left\{u\left(t_{1}\right)\right\}}\right], \\
& 0=\left.\frac{d n_{1}(t)}{d t}\right|_{t=t_{2}}=\lambda\left[\frac{c\left(t_{2}\right) \exp \left\{u\left(t_{2}-\tau\left(t_{2}\right)\right)\right\} \exp \left\{n_{2}\left(t_{2}-\tau\left(t_{2}\right)\right)-n_{1}\left(t_{2}\right)\right\}}{m \exp \left\{n_{2}\left(t_{2}-\tau\left(t_{2}\right)\right)\right\}+\exp \left\{u\left(t_{2}-\tau\left(t_{2}\right)\right)\right\}}\right. \\
& \left.-\left(D\left(t_{2}\right)+v_{1}\left(t_{2}\right)\right)\right] \\
& 0=\left.\frac{d n_{2}(t)}{d t}\right|_{t=t_{3}}=\lambda\left[D\left(t_{3}\right) \exp \left\{n_{1}\left(t_{3}\right)-n_{2}\left(t_{3}\right)\right\}-v_{2}\left(t_{3}\right)\right]
\end{aligned}
$$


or

$$
\begin{gathered}
0=r\left(t_{1}\right)-a\left(t_{1}\right) \exp \left\{u\left(t_{1}\right)\right\}-\frac{b\left(t_{1}\right) \exp \left\{n_{2}\left(t_{1}\right)\right\}}{m \exp \left\{n_{2}\left(t_{1}\right)\right\}+\exp \left\{u\left(t_{1}\right)\right\}}, \\
0=\frac{c\left(t_{2}\right) \exp \left\{u\left(t_{2}-\tau\left(t_{2}\right)\right)\right\} \exp \left\{n_{2}\left(t_{2}-\tau\left(t_{2}\right)\right)-n_{1}\left(t_{2}\right)\right\}}{m \exp \left\{n_{2}\left(t_{2}-\tau\left(t_{2}\right)\right)\right\}+\exp \left\{u\left(t_{2}-\tau\left(t_{2}\right)\right)\right\}}-\left(D\left(t_{2}\right)+v_{1}\left(t_{2}\right)\right), \\
0=D\left(t_{3}\right) \exp \left\{n_{1}\left(t_{3}\right)-n_{2}\left(t_{3}\right)\right\}-v_{2}\left(t_{3}\right) .
\end{gathered}
$$

From (2.16), one has

$$
\frac{c\left(t_{2}\right) \exp \left\{u\left(t_{2}-\tau\left(t_{2}\right)\right)\right\} \exp \left\{n_{2}\left(t_{2}-\tau\left(t_{2}\right)\right)\right\}}{m \exp \left\{n_{2}\left(t_{2}-\tau\left(t_{2}\right)\right)\right\}+\exp \left\{u\left(t_{2}-\tau\left(t_{2}\right)\right)\right\}}=\exp \left\{n_{1}\left(t_{2}\right)\right\}\left(D\left(t_{2}\right)+v_{1}\left(t_{2}\right)\right) .
$$

From (2.17), one has

$$
D\left(t_{3}\right) \exp \left\{n_{1}\left(t_{3}\right)\right\}=\exp \left\{n_{2}\left(t_{3}\right)\right\} v_{2}\left(t_{3}\right),
$$

which implies that

$$
\exp \left\{n_{2}\left(t_{3}\right)\right\} \leq \frac{D^{u}}{v_{2}^{l}} \exp \left\{n_{1}\left(t_{3}\right)\right\}
$$

From (2.15), one has

$$
r\left(t_{1}\right)-a\left(t_{1}\right) \exp \left\{u\left(t_{1}\right)\right\}>0,
$$

therefore,

$$
u\left(t_{1}\right) \leq \ln \frac{r^{u}}{a^{l}} .
$$

By using (2.20) and (2.22), from (2.19), it follows that

$$
\begin{aligned}
\exp \{ & \left.n_{1}\left(t_{2}\right)\right\}\left(D\left(t_{2}\right)+v_{1}\left(t_{2}\right)\right) \\
& =\frac{c\left(t_{2}\right) \exp \left\{u\left(t_{2}-\tau\left(t_{2}\right)\right)\right\} \exp \left\{n_{2}\left(t_{2}-\tau\left(t_{2}\right)\right)\right\}}{m \exp \left\{n_{2}\left(t_{2}-\tau\left(t_{2}\right)\right)\right\}+\exp \left\{u\left(t_{2}-\tau\left(t_{2}\right)\right)\right\}} \\
& \leq \frac{c\left(t_{2}\right) \exp \left\{u\left(t_{1}\right)\right\} \exp \left\{n_{2}\left(t_{3}\right)\right\}}{m \exp \left\{n_{2}\left(t_{3}\right)\right\}+\exp \left\{u\left(t_{1}\right)\right\}} \leq \frac{c\left(t_{2}\right)\left(r^{u} / a^{l}\right)\left(D^{u} / v_{2}^{l}\right) \exp \left\{n_{1}\left(t_{2}\right)\right\}}{m\left(D^{u} / v_{2}^{l}\right) \exp \left\{n_{1}\left(t_{2}\right)\right\}+r^{u} / a^{l}},
\end{aligned}
$$

which implies

$$
\exp \left\{n_{1}\left(t_{2}\right)\right\}\left(D^{l}+v_{1}^{l}\right) \leq \frac{c^{u}\left(r^{u} / a^{l}\right)\left(D^{u} / v_{2}^{l}\right) \exp \left\{n_{1}\left(t_{2}\right)\right\}}{m\left(D^{u} / v_{2}^{l}\right) \exp \left\{n_{1}\left(t_{2}\right)\right\}+r^{u / a^{l}}} .
$$

Therefore,

$$
\left(D^{l}+v_{1}^{l}\right)\left(m \frac{D^{u}}{v_{2}^{l}} \exp \left\{n_{1}\left(t_{2}\right)\right\}+\frac{r^{u}}{a^{l}}\right) \leq c^{u} \frac{r^{u}}{a^{l}} \frac{D^{u}}{v_{2}^{l}} .
$$


Solving the above inequality, one obtains

$$
\exp \left\{n_{1}\left(t_{2}\right)\right\} \leq \frac{\left(r^{u} / a^{l} v_{2}^{l}\right)\left[c^{u} D^{u}-v_{2}^{l}\left(D^{l}+v_{1}^{l}\right)\right]}{\left(D^{l}+v_{1}^{l}\right) m\left(D^{u} / v_{2}^{l}\right)}:=B_{1},
$$

therefore,

$$
n_{1}\left(t_{2}\right) \leq \rho_{2}
$$

where $\rho_{2}=\ln B_{1}$. Again, from (2.20), one has

$$
\exp \left\{n_{2}\left(t_{3}\right)\right\} \leq \frac{D^{u}}{v_{2}^{l}} \exp \left\{n_{1}\left(t_{3}\right)\right\} \leq \frac{D^{u}}{v_{2}^{l}} \exp \left\{n_{1}\left(t_{2}\right)\right\} \leq \frac{D^{u}}{v_{2}^{l}} B_{1} .
$$

And so,

$$
n_{2}\left(t_{3}\right) \leq \rho_{3}
$$

where $\rho_{3}=\ln \left(D^{u} / v_{2}^{l}\right) B_{1}$.

On the other hand, since $x \in X$, there also exist $t_{i}^{\prime} \in[0, \omega]$ such that

$$
u\left(t_{1}^{\prime}\right)=\min _{t \in[0, \omega]} u(t), \quad n_{1}\left(t_{2}^{\prime}\right)=\min _{t \in[0, \omega]} n_{1}(t), \quad n_{2}\left(t_{3}^{\prime}\right)=\min _{t \in[0, \omega]} n_{2}(t) .
$$

Then one has

$$
\begin{aligned}
& 0=\left.\frac{d u(t)}{d t}\right|_{t=t_{1}^{\prime}}=\lambda\left[r\left(t_{1}^{\prime}\right)-a\left(t_{1}^{\prime}\right) \exp \left\{u\left(t_{1}^{\prime}\right)\right\}-\frac{b\left(t_{1}^{\prime}\right) \exp \left\{n_{2}\left(t_{1}^{\prime}\right)\right\}}{m \exp \left\{n_{2}\left(t_{1}^{\prime}\right)\right\}+\exp \left\{u\left(t_{1}^{\prime}\right)\right\}}\right], \\
& 0=\left.\frac{d n_{1}(t)}{d t}\right|_{t=t_{2}^{\prime}}=\lambda\left[\frac{c\left(t_{2}^{\prime}\right) \exp \left\{u\left(t_{2}^{\prime}-\tau\left(t_{2}^{\prime}\right)\right)\right\} \exp \left\{n_{2}\left(t_{2}^{\prime}-\tau\left(t_{2}^{\prime}\right)\right)-n_{1}\left(t_{2}^{\prime}\right)\right\}}{m \exp \left\{n_{2}\left(t_{2}^{\prime}-\tau\left(t_{2}^{\prime}\right)\right)\right\}+\exp \left\{u\left(t_{2}^{\prime}-\tau\left(t_{2}^{\prime}\right)\right)\right\}}\right. \\
& \left.-\left(D\left(t_{2}^{\prime}\right)+v_{1}\left(t_{2}^{\prime}\right)\right)\right] \\
& 0=\left.\frac{d n_{2}(t)}{d t}\right|_{t=t_{3}^{\prime}}=\lambda\left[D\left(t_{3}^{\prime}\right) \exp \left\{n_{1}\left(t_{3}^{\prime}\right)-n_{2}\left(t_{3}^{\prime}\right)\right\}-v_{2}\left(t_{3}^{\prime}\right)\right]
\end{aligned}
$$

or

$$
\begin{gathered}
0=r\left(t_{1}^{\prime}\right)-a\left(t_{1}^{\prime}\right) \exp \left\{u\left(t_{1}^{\prime}\right)\right\}-\frac{b\left(t_{1}^{\prime}\right) \exp \left\{n_{2}\left(t_{1}^{\prime}\right)\right\}}{m \exp \left\{n_{2}\left(t_{1}^{\prime}\right)\right\}+\exp \left\{u\left(t_{1}^{\prime}\right)\right\}}, \\
0=\frac{c\left(t_{2}^{\prime}\right) \exp \left\{u\left(t_{2}^{\prime}-\tau\left(t_{2}^{\prime}\right)\right)\right\} \exp \left\{n_{2}\left(t_{2}^{\prime}-\tau\left(t_{2}^{\prime}\right)\right)-n_{1}\left(t_{2}^{\prime}\right)\right\}}{m \exp \left\{n_{2}\left(t_{2}^{\prime}-\tau\left(t_{2}^{\prime}\right)\right)\right\}+\exp \left\{u\left(t_{2}^{\prime}-\tau\left(t_{2}^{\prime}\right)\right)\right\}}-\left(D\left(t_{2}^{\prime}\right)+v_{1}\left(t_{2}^{\prime}\right)\right), \\
0=D\left(t_{3}^{\prime}\right) \exp \left\{n_{1}\left(t_{3}^{\prime}\right)-n_{2}\left(t_{3}^{\prime}\right)\right\}-v_{2}\left(t_{3}^{\prime}\right) .
\end{gathered}
$$

From (2.33), one has

$$
\frac{c\left(t_{2}^{\prime}\right) \exp \left\{u\left(t_{2}^{\prime}-\tau\left(t_{2}^{\prime}\right)\right)\right\} \exp \left\{n_{2}\left(t_{2}^{\prime}-\tau\left(t_{2}^{\prime}\right)\right)\right\}}{m \exp \left\{n_{2}\left(t_{2}^{\prime}-\tau\left(t_{2}^{\prime}\right)\right)\right\}+\exp \left\{u\left(t_{2}^{\prime}-\tau\left(t_{2}^{\prime}\right)\right)\right\}}=\exp \left\{n_{1}\left(t_{2}^{\prime}\right)\right\}\left(D\left(t_{2}^{\prime}\right)+v_{1}\left(t_{2}^{\prime}\right)\right) .
$$


From (2.34), one has

$$
D\left(t_{3}^{\prime}\right) \exp \left\{n_{1}\left(t_{3}^{\prime}\right)\right\}=\exp \left\{n_{2}\left(t_{3}^{\prime}\right)\right\} v_{2}\left(t_{3}^{\prime}\right)
$$

which implies that

$$
\exp \left\{n_{2}\left(t_{3}^{\prime}\right)\right\} \geq \frac{D^{l}}{v_{2}^{u}} \exp \left\{n_{1}\left(t_{3}^{\prime}\right)\right\} \geq \frac{D^{l}}{v_{2}^{u}} \exp \left\{n_{1}\left(t_{2}^{\prime}\right)\right\} .
$$

From (2.32), one has

$$
r\left(t_{1}^{\prime}\right)-a\left(t_{1}^{\prime}\right) \exp \left\{u\left(t_{1}^{\prime}\right)\right\}-\frac{b\left(t_{1}^{\prime}\right)}{m} \leq 0,
$$

therefore,

$$
a\left(t_{1}^{\prime}\right) \exp \left\{u\left(t_{1}^{\prime}\right)\right\} \geq r\left(t_{1}^{\prime}\right)-\frac{b\left(t_{1}^{\prime}\right)}{m} .
$$

By using Theorem 2.2(i), one has

$$
u\left(t_{1}^{\prime}\right) \geq \ln \frac{m r^{l}-b^{u}}{m a^{u}} .
$$

By using (2.36), (2.37), and (2.40), from (2.35), it follows that

$$
\begin{aligned}
\exp \{ & \left.n_{1}\left(t_{2}^{\prime}\right)\right\}\left(D\left(t_{2}^{\prime}\right)+v_{1}\left(t_{2}^{\prime}\right)\right) \\
& =\frac{c\left(t_{2}^{\prime}\right) \exp \left\{u\left(t_{2}^{\prime}-\tau\left(t_{2}^{\prime}\right)\right)\right\} \exp \left\{n_{2}\left(t_{2}^{\prime}-\tau\left(t_{2}^{\prime}\right)\right)\right\}}{m \exp \left\{n_{2}\left(t_{2}^{\prime}-\tau\left(t_{2}^{\prime}\right)\right)\right\}+\exp \left\{u\left(t_{2}^{\prime}-\tau\left(t_{2}^{\prime}\right)\right)\right\}} \\
& \geq \frac{c\left(t_{2}\right) \exp \left\{u\left(t_{1}^{\prime}\right)\right\} \exp \left\{n_{2}\left(t_{2}^{\prime}-\tau\left(t_{2}^{\prime}\right)\right)\right\}}{m \exp \left\{n_{2}\left(t_{2}^{\prime}-\tau\left(t_{2}^{\prime}\right)\right)\right\}+\exp \left\{u\left(t_{1}^{\prime}\right)\right\}} \\
& \geq \frac{c^{l}\left(\left(m r^{l}-b^{u}\right) / m a^{u}\right)\left(D^{l} / v_{2}^{u}\right) \exp \left\{n_{1}\left(t_{2}^{\prime}\right)\right\}}{m\left(D^{l} / v_{2}^{u}\right) \exp \left\{n_{1}\left(t_{2}^{\prime}\right)\right\}+\left(m r^{l}-b^{u}\right) / m a^{u}},
\end{aligned}
$$

which implies

$$
\exp \left\{n_{1}\left(t_{2}^{\prime}\right)\right\}\left(D^{u}+v_{1}^{u}\right) \geq \frac{c^{l}\left(\left(m r^{l}-b^{u}\right) / m a^{u}\right)\left(D^{l} / v_{2}^{u}\right) \exp \left\{n_{1}\left(t_{2}^{\prime}\right)\right\}}{m\left(D^{l} / v_{2}^{u}\right) \exp \left\{n_{1}\left(t_{2}^{\prime}\right)\right\}+\left(m r^{l}-b^{u}\right) / m a^{u}},
$$

therefore,

$$
\left(D^{u}+v_{1}^{u}\right)\left(m \frac{D^{l}}{v_{2}^{u}} \exp \left\{n_{1}\left(t_{2}^{\prime}\right)\right\}+\frac{m r^{l}-b^{u}}{m a^{u}}\right) \geq c^{l} \frac{m r^{l}-b^{u}}{m a^{u}} \frac{D^{l}}{v_{2}^{u}} .
$$

By using Theorem 2.2(ii), solving the above inequality, one obtains

$$
\exp \left\{n_{1}\left(t_{2}^{\prime}\right)\right\} \geq \frac{\left(\left(m r^{l}-b^{u}\right) / m a^{u} v_{2}^{u}\right)\left[c^{l} D^{l}-v_{2}^{u}\left(D^{u}+v_{1}^{u}\right)\right]}{\left(D^{u}+v_{1}^{u}\right) m\left(D^{l} / v_{2}^{u}\right)}:=B_{1}^{\prime},
$$


162 A stage-structured ratio-dependent predator-prey model

therefore,

$$
n_{1}\left(t_{2}\right) \geq \rho_{2}^{*}
$$

where $\rho_{2}^{*}=\ln B_{1}^{\prime}$. Again, from (2.37), one has

$$
\exp \left\{n_{2}\left(t_{3}^{\prime}\right)\right\} \geq \frac{D^{l}}{v_{2}^{u}} \exp \left\{n_{1}\left(t_{3}^{\prime}\right)\right\} \geq \frac{D^{l}}{v_{2}^{u}} \exp \left\{n_{1}\left(t_{2}^{\prime}\right)\right\} \geq \frac{D^{l}}{v_{2}^{u}} B_{1}^{\prime} .
$$

And so,

$$
n_{2}\left(t_{3}\right) \geq \rho_{3}^{*}
$$

where $\rho_{3}^{*}=\ln \left(D^{l} / v_{2}^{u}\right) B_{1}^{\prime}$. It follows from (2.22) and (2.40) that

$$
\max _{t \in[0, \omega]}|u(t)| \leq \max \left\{\left|\ln \frac{r^{u}}{a^{l}}\right|,\left|\ln \frac{m r^{l}-b^{u}}{m a^{u}}\right|\right\}:=R_{1} .
$$

And from (2.27), (2.29), (2.45), and (2.47), one has

$$
\begin{aligned}
& \max _{t \in[0, \omega]}\left|n_{1}(t)\right| \leq \max \left\{\left|\rho_{2}\right|,\left|\rho_{2}^{*}\right|\right\}:=R_{2}, \\
& \max _{t \in[0, \omega]}\left|n_{2}(t)\right| \leq \max \left\{\left|\rho_{3}\right|,\left|\rho_{3}^{*}\right|\right\}:=R_{3} .
\end{aligned}
$$

Clearly, $R_{i}(i=1,2,3)$ are independent of the choice of $\lambda$. Under the assumptions in Theorem 2.2, it is easy to show that the system of algebraic equations

$$
\begin{gathered}
\bar{r}-\bar{a} u-\frac{\bar{b} n_{2}}{m n_{2}+u}=0, \\
\bar{c} \cdot \frac{u}{m n_{2}+u} \cdot \frac{n_{2}}{n_{1}}-\left(\bar{D}+\bar{v}_{1}\right)=0, \\
\bar{D} \frac{n_{1}}{n_{2}}-\bar{v}_{2}=0
\end{gathered}
$$

has a unique solution $\left(u^{*}, x_{1}^{*}, x_{2}^{*}\right)^{T} \in \int R_{+}^{3}$ with $u^{*}>0, x_{i}^{*}>0, i=1,2$. Take $B=R_{1}+R_{2}+$ $R_{3}+R_{0}$, where $R_{0}>0$ is taken sufficiently large such that $\left\|\left(\ln \left\{u^{*}\right\}, \ln \left\{v_{1}^{*}\right\}, \ln \left\{v_{2}^{*}\right\}\right)^{T}\right\|=$ $\left|\ln \left\{u^{*}\right\}\right|+\left|\ln \left\{v_{1}^{*}\right\}\right|+\left|\ln \left\{v_{2}^{*}\right\}\right|<R_{0}$, and define

$$
\Omega=\left\{x(t)=\left(u(t), n_{1}(t), n_{2}(t)\right)^{T} \in X:\|x\|<R\right\} .
$$

It is clear that $\Omega$ verifies the requirement in Lemma 2.1(a). When $x \in \partial \Omega \cap \operatorname{Ker} L=\partial \Omega \cap$ $R^{3}, x$ is a constant vector in $R^{3}$ with $\|x\|=B$. Then

$$
Q N x=\left(\begin{array}{c}
\bar{r}-\bar{a} \exp \{u\}-\frac{\bar{b} \exp \left\{n_{2}\right\}}{m \exp \left\{n_{2}\right\}+\exp \{u\}} \\
\bar{c} \frac{\exp \{u\}}{m n_{2}+\exp \{u\}} \exp \left\{n_{1}-n_{2}\right\}-\left(\bar{D}+\bar{v}_{1}\right) \\
\bar{D} \exp \left\{n_{1}-n_{2}\right\}-\bar{v}_{2}
\end{array}\right) \neq 0 .
$$


Furthermore, in view of the assumptions in Theorem 2.2, direct calculation produces

$$
\operatorname{deg}\{J Q N, \Omega \cap \operatorname{Ker} L, 0\}=\operatorname{sgn}\left(\frac{-\bar{D} \bar{c} m \exp \left\{2 u^{*}+n_{2}^{*}\right\}}{\left(m \exp \left\{n_{2}^{*}\right\}+\exp \left\{u^{*}\right\}\right)^{2}}\right)=-1 \neq 0 .
$$

Here $J$ can be the identity mapping since $\operatorname{Im} P=\operatorname{Ker} L$. By now we have proved that $\Omega$ verifies all the requirements in Lemma 2.1. Hence, (2.4) has at least one solution $\left(u^{*}(t), n_{1}^{*}(t), n_{2}^{*}(t)\right)^{T}$ in $\operatorname{Dom} L \cap \bar{\Omega}$. Set $x^{*}(t)=\exp \left\{u^{*}(t)\right\}, y_{1}^{*}(t)=\exp \left\{n_{1}^{*}(t)\right\}, y_{2}^{*}(t)=$ $\exp \left\{n_{2}^{*}(t)\right\}$, then by the medium of (2.3), we know that $\left(x^{*}(t), y_{1}^{*}(t), y_{2}^{*}(t)\right)^{T}$ is an $\omega$ periodic solution of (1.4) with strictly positive components. This completes the proof of Theorem 2.2.

Remark 2.3. From the proof of Theorem 2.2, one can observe that Theorem 2.2 remains valid if discrete delay in (1.4) is replaced by state-dependent delay $\tau\left(t, x(t), y_{1}(t), y_{2}(t)\right)$ or constant delay $\tau(t) \equiv \tau$. We note that discrete delay has no influence on the existence of positive periodic solution of the system.

\section{Permanence and stability of periodic solution}

This section is devoted to study the stability of system (1.4) under the assumption $\tau(t) \equiv 0$, throughout this section, we assume that

$$
\text { (H) } \tau(t) \equiv 0 \text { in system (1.4) }
$$

We will now proceed to derive sufficient conditions to guarantee the permanence of system (1.4). To this end, we first obtain certain upper bound estimates for positive solutions of system (1.4) with initial conditions (1.5).

LEMma 3.1. Let $\left(x(t), y_{1}(t), y_{2}(t)\right)$ be any positive solution to system (1.4) with initial conditions (1.5). Then there exist a $T>0$ and an $M>0$ such that $x(t)<M, y_{1}(t)<M$, and $y_{2}(t)<M$ for all $t>T$.

Proof. It follows from the first equation of system (1.4) that

$$
\frac{d x(t)}{d t} \leq x(t)\left(r^{u}-a^{l} x(t)\right)
$$

From this, by using the comparison theorem, one could deduce that there exists a $T>0$ such that

$$
x(t)<\frac{r^{u}}{a^{l}}+\varepsilon:=M_{0},
$$

where $\varepsilon$ is an arbitrary small positive number. 
Now let $V(t)=\left(c^{u} / b^{l}\right) x(t)+y_{1}(t)$, calculating the derivative of $V(t)$ along the positive solution of system (1.4), then for $t>T$,

$$
\begin{aligned}
V^{\prime}(t) & =\frac{c^{u}}{b^{l}}\left(x(t)(r(t)-a(t) x(t))-\frac{b(t) x(t) y_{2}(t)}{m y_{2}(t)+x(t)}\right)+\frac{c(t) x(t) y_{2}(t)}{m y_{2}(t)+x(t)}-\left(D(t)+v_{1}(t)\right) y_{1}(t) \\
& \leq \frac{c^{u} r^{u}}{b^{l}} x(t)-\left(D^{l}+v_{1}^{l}\right) y_{1}(t)=-\left(D^{l}+v_{1}^{l}\right) V(t)+\frac{c^{u}}{b^{l}}\left(D^{l}+v_{1}^{l}+r^{u}\right) M_{0} .
\end{aligned}
$$

Noticing that $x(t)<M_{0}$ for $t>T$, from the definition of $V(t)$, one can deduce that there exists an $M_{1}>0$ such that

$$
y_{1}(t)<M_{1} \quad \text { for } t \geq T
$$

From (3.4) and the third equation of system (1.4), by using the comparison theorem, one could deduce there exists an $M_{2}>0$ such that

$$
y_{2}(t)<M_{2} \quad \text { for } t \geq T \text {. }
$$

Take $M=\max \left\{M_{0}, M_{1}, M_{2}\right\}$, then $x(t)<M, y_{1}(t)<M$, and $y_{2}(t)<M$ for all $t>T$. This ends the proof of Lemma 3.1.

Theorem 3.2. In addition to condition $(H)$, assume that the conditions of Theorem 2.2 hold. Then system (1.4) is permanent.

Proof. Let $\left(x(t), y_{1}(t), y_{2}(t)\right)$ be any positive solution to system (1.4) with initial conditions (1.5). Under the assumption $m r^{l}>b^{u}$, one has

$$
\frac{d x}{d t} \geq x(t)\left(r^{l}-\frac{b^{u}}{m}-a^{l} x(t)\right) .
$$

From this, one has

$$
\lim _{t \rightarrow+\infty} \inf x(t) \geq \frac{r^{l} m-b^{u}}{m a^{u}}:=x^{*}
$$

and so, there exists a $T_{1}>0$ such that

$$
x(t) \geq \frac{x^{*}}{2}:=m_{1} \quad \text { for } t \geq T_{1} .
$$

By using (3.8), from the second and third equations of system (1.4), one has

$$
\begin{gathered}
\frac{d y_{1}(t)}{d t} \geq \frac{c^{l}\left(x^{*} / 2\right) y_{2}(t)}{m y_{2}(t)+x^{*} / 2}-\left(D^{u}+v_{1}^{u}\right) y_{1}(t), \\
\frac{d y_{2}(t)}{d t} \geq D^{l} y_{1}(t)-v_{2}^{u} y_{2}(t) .
\end{gathered}
$$


Now we consider the system

$$
\begin{gathered}
\frac{d y_{1}(t)}{d t}=\frac{c^{l}\left(x^{*} / 2\right) y_{2}(t)}{m y_{2}(t)+x^{*} / 2}-\left(D^{u}+v_{1}^{u}\right) y_{1}(t), \\
\frac{d y_{2}(t)}{d t}=D^{l} y_{1}(t)-v_{2}^{u} y_{2}(t) .
\end{gathered}
$$

By using Lemma 3.1, similar to the analysis of [12, page 69], under the assumption of Theorem 3.2, one could deduce that the unique positive equilibrium $\left(y_{1}^{*}, y_{2}^{*}\right)=$ $\left(\left(v_{2}^{u} / D^{l}\right) y_{2}^{*},\left(c^{l} D^{l}-\left(D^{u}+v_{1}^{u}\right) v_{2}^{u}\right) x^{*} /\left(D^{u}+v_{1}^{u}\right) v_{2}^{u} m\right)$ of the above system is globally attractive. And so, by using the comparison theorem, one could deduce that there exists a $T_{2}>T_{1}$ such that

$$
y_{1}(t) \geq \frac{y_{1}^{*}}{2}:=m_{2}, \quad y_{2}(t) \geq \frac{y_{2}^{*}}{2}:=m_{3}
$$

Now we take $T=T_{2}$, then

$$
x(t) \geq m_{1}, \quad y_{1}(t) \geq m_{2}, \quad y_{2}(t) \geq m_{3}, \quad \text { for } t \geq T
$$

This ends the proof of Theorem 3.2.

THeOREM 3.3. In addition to the conditions of Theorem 3.2, assume further that

$$
\min \left\{a(t), v_{2}(t)\right\}>\frac{b(t) M+c(t) m M^{2}}{\left(m m_{3}+m_{1}\right)^{2}},
$$

where $M, m_{3}$, and $m_{1}$ are defined by Lemma 3.1 and (3.12), respectively. Then system (1.4) with initial conditions (1.5) has a unique globally attractive positive periodic solution.

Proof. From Theorem 3.2, (1.4) has at least one positive periodic solution. Let $\left(x^{*}(t)\right.$, $\left.y_{1}^{*}(t), y_{2}^{*}(t)\right)^{T}$ be a positive $\omega$-periodic solution of system (1.4) with initial conditions (1.5). Suppose that $\left(x(t), y_{1}(t), y_{2}(t)\right)^{T}$ is any positive solution of system (1.4). Let

$$
V(t)=\left|\ln x(t)-\ln x^{*}(t)\right|+\left|y_{1}(t)-y_{1}^{*}(t)\right|+\left|y_{2}(t)-y_{2}^{*}(t)\right|
$$

A direct calculation of the right derivative $D^{+} V(t)$ along the solution of (1.4) produces

$$
\begin{aligned}
& D^{+} V(t)= \operatorname{sgn}\left(x(t)-x^{*}(t)\right)\left[-a(t)\left(x(t)-x^{*}(t)\right)-\left(\frac{b(t) y_{2}(t)}{m y_{2}(t)+x(t)}-\frac{b(t) y_{2}^{*}(t)}{m y_{2}^{*}(t)+x^{*}(t)}\right)\right] \\
&+\operatorname{sgn}\left(y_{1}(t)-y_{1}^{*}(t)\right)\left[\left(\frac{c(t) x(t) y_{2}(t)}{m y_{2}(t)+x(t)}-\frac{c(t) x^{*}(t) y_{2}^{*}(t)}{m y_{2}^{*}(t)+x_{2}^{*}(t)}\right)\right. \\
&\left.-\left(D(t)+v_{1}(t)\right)\left(y_{1}(t)-y_{1}^{*}(t)\right)\right] \\
&+\operatorname{sgn}\left(y_{2}(t)-y_{2}^{*}(t)\right)\left[D(t)\left(y_{1}(t)-y_{1}^{*}(t)\right)-v_{2}(t)\left(y_{2}(t)-y_{2}^{*}(t)\right)\right] .
\end{aligned}
$$


Note that

$$
y_{2}^{*}(t) x(t)-y_{2}(t) x^{*}(t)=x(t)\left(y_{2}^{*}(t)-y_{2}(t)\right)+y_{2}(t)\left(x(t)-x^{*}(t)\right) .
$$

So, for $t \geq T$ (here $T$ is defined by Theorem 3.2), one has

$$
\begin{aligned}
& \operatorname{sgn}(\left.x(t)-x^{*}(t)\right)\left(-\frac{b(t) y_{2}(t)}{m y_{2}(t)+x(t)}+\frac{b(t) y_{2}^{*}(t)}{m y_{2}^{*}(t)+x^{*}(t)}\right) \\
& \leq \frac{b(t) x(t)\left|y_{2}(t)-y_{2}^{*}(t)\right|}{\left(m y_{2}(t)+x(t)\right)\left(m y_{2}^{*}(t)+x^{*}(t)\right)}+\frac{b(t) y_{2}(t)\left|x(t)-x^{*}(t)\right|}{\left(m y_{2}(t)+x(t)\right)\left(m y_{2}^{*}(t)+x^{*}(t)\right)} \\
& \leq \frac{b(t) M\left|y_{2}(t)-y_{2}^{*}(t)\right|}{\left(m m_{3}+m_{1}\right)^{2}}+\frac{b(t) M\left|x(t)-x^{*}(t)\right|}{\left(m m_{3}+m_{1}\right)^{2}}, \\
& \operatorname{sgn}\left(y_{1}(t)-y_{1}^{*}(t)\right)\left(\frac{c(t) x(t) y_{2}(t)}{m y_{2}(t)+x(t)}-\frac{c(t) x^{*}(t) y_{2}^{*}(t)}{m y_{2}^{*}(t)+x_{2}^{*}(t)}\right) \\
& \quad \leq \frac{c(t) m y_{2}(t) y_{2}^{*}(t)\left|x(t)-x^{*}(t)\right|}{\left(m y_{2}^{*}(t)+x_{2}^{*}(t)\right)\left(m y_{2}(t)+x(t)\right)}+\frac{c(t) m x(t) x^{*}(t)\left|y_{2}(t)-y_{2}^{*}(t)\right|}{\left(m y_{2}^{*}(t)+x_{2}^{*}(t)\right)\left(m y_{2}(t)+x(t)\right)} \\
& \leq \frac{c(t) m M^{2}\left|x(t)-x^{*}(t)\right|}{\left(m m_{3}+m_{1}\right)^{2}}+\frac{c(t) m M^{2}\left|y_{2}(t)-y_{2}^{*}(t)\right|}{\left(m m_{3}+m_{1}\right)^{2}},
\end{aligned}
$$

where $M, m_{3}$, and $m_{1}$ are defined by Lemma 3.1 and (3.12), respectively. Then when $t>T$ from (3.17), one has

$$
\begin{aligned}
D^{+} V(t) \leq & -\left(a(t)-\frac{b(t) M}{\left(m m_{3}+m_{1}\right)^{2}}-\frac{c(t) m M^{2}}{\left(m m_{3}+m_{1}\right)^{2}}\right)\left|x(t)-x^{*}(t)\right| \\
& -v_{1}(t)\left|y_{1}(t)-y_{1}^{*}(t)\right| \\
& -\left(v_{2}(t)-\frac{c(t) m M^{2}}{\left(m m_{3}+m_{1}\right)^{2}}-\frac{b(t) M}{\left(m m_{3}+m_{1}\right)^{2}}\right)\left|y_{2}(t)-y_{2}^{*}(t)\right| .
\end{aligned}
$$

From the above inequality, combined with the condition of the Theorem 3.2, it follows that there exists a positive constant $\mu>0$ such that

$$
D^{+} V(t) \leq-\mu\left[\left|x(t)-x^{*}(t)\right|+\sum_{i=1}^{2}\left|y_{i}(t)-y_{i}^{*}(t)\right|\right], \quad t>T .
$$

Integrating on both sides of (3.19) from $T$ to $t$ produces

$$
V(t)+\mu \int_{T}^{t}\left[\left|x(s)-x^{*}(s)\right|+\sum_{i=1}^{2}\left|y_{i}(s)-y_{i}^{*}(s)\right|\right] d s \leq V(T)<+\infty, \quad t \geq T .
$$

Then

$$
\int_{T}^{t}\left[\left|x(s)-x^{*}(s)\right|+\sum_{i=1}^{2}\left|y_{i}(s)-y_{i}^{*}(s)\right|\right] d s \leq \mu^{-1} V(T)<+\infty, \quad t \geq T .
$$


And hence, $\left|x(t)-x^{*}(t)\right|+\sum_{i=1}^{2}\left|y_{i}(t)-y_{i}^{*}(t)\right| \in L^{1}([T,+\infty))$. The boundedness of $x^{*}(t)$ and $y_{i}^{*}(t)(i=1,2)$ and the ultimate boundedness of $x(t)$ and $y_{i}(t)(i=1,2)$ imply that $x(t), y_{i}(t), x^{*}(t)$, and $y_{i}^{*}(t)(i=1,2)$ all have bounded derivatives for $t>T$ (from the equations satisfied by them). Then it follows that $\left|x(t)-x^{*}(t)\right|+\sum_{i=1}^{2}\left|y_{i}(t)-y_{i}^{*}(t)\right|$ is uniformly continuous on $[T,+\infty)$. And so

$$
\lim _{t \rightarrow+\infty}\left(\left|x(t)-x^{*}(t)\right|+\sum_{i=1}^{2}\left|y_{i}(t)-y_{i}^{*}(t)\right|\right)=0 .
$$

The proof is completed.

The following numerical example shows the feasibility of our main result.

Example 3.4. Consider the following nonautonomous stage-structured ratio-dependent predator-prey system:

$$
\begin{gathered}
\frac{d x(t)}{d t}=x(t)(12+3 \sin t-(2+\cos 2 t) x(t))-\frac{(4-\sin t) x(t) y_{2}(t)}{2 y_{2}(t)+x(t)} \\
\frac{d y_{1}(t)}{d t}=\frac{3 x(t-\tau(t)) y_{2}(t-\tau(t))}{2 y_{2}(t-\tau(t))+x(t-\tau(t))}-\left(\frac{1}{2}+\left(\frac{1}{2}+\frac{\cos t}{2}\right)\right) y_{1}(t) \\
\frac{d y_{2}(t)}{d t}=\frac{1}{2} y_{1}(t)-\frac{1}{2} y_{2}(t)
\end{gathered}
$$

where $\tau(t) \geq 0$ is a continuous $2 \pi$-periodic function. In this case, corresponding to system (1.4), one has $r(t)=12+3 \sin t, a(t)=2+\cos 2 t, b(t)=4-\sin t, c(t)=3, D(t)=1 / 2$, $v_{1}(t)=1 / 2+\cos t / 2, v_{2}(t)=1 / 2, m=2$, and so

$$
\begin{gathered}
m r^{l}=2 \times 9>b^{u}=5, \\
c^{l} D^{l}=3 \times \frac{1}{2}=\frac{3}{2}>\frac{1}{2}\left(\frac{1}{2}+1\right)=\frac{3}{4}=v_{2}^{u}\left(D^{u}+v_{1}^{u}\right) .
\end{gathered}
$$

By Theorem 2.2, system (3.23) has at least one positive $2 \pi$-periodic solution. Also, if we further assume that $\tau(t) \equiv 0$, then system (3.23) is permanent.

We end this paper by some remarks. In this paper, a set of sufficient conditions has been derived ensuring the existence of periodic solution of the periodic system (1.4) by using the theory of coincidence degree and developing the analysis technique of [12]. To the best of our knowledge, it is the first time to consider system (1.4). If all the parameters in (1.4), in particular, are restricted to be positive constants, then the conditions in Theorem 2.2 are sufficient to guarantee the existence of a positive equilibrium (for more details, one could refer to [26]), that is to say, in this case, the positive periodic solution obtained in Theorem 2.2 may degenerate to a trivial periodic solution. By Theorem 2.2, we see that if the prey intrinsic growth rate $(r(t))$, the conversion rate of nutrients into the reproduction of the predator $(k(t)=c(t) / b(t))$, and the capture rate of the predator $(b(t))$ are high, and the death rate of both the immature $\left(v_{1}(t)\right)$ and mature $\left(v_{2}(t)\right)$ predators is low, then system (1.4) admits at least one positive periodic solution. For the system 
without delay, by constructing a suitable Lyapunov function, sufficient conditions are derived to ensure the global attractivity of the positive periodic solution. These criteria are of great interest in many applications such as biomathematics and computation.

\section{Acknowledgments}

This work is supported by the National Natural Science Foundation of China (Tian Yuan Foundation) (10426010), the Foundation of Science and Technology of Fujian Province for Young Scholars (2004J0002), the Foundation of Fujian Education Bureau (JA04156), and the Foundation of Developing Science and Technology of Fuzhou University (2003QX-21). I am very grateful to the two referees for their very helpful suggestions and careful reading of the manuscript.

\section{References}

[1] R. Arditi and L. R. Ginzburg, Coupling in predator-prey dynamics: ratio-dependence, J. Theoret. Biol. 139 (1989), 311-326.

[2] R. Arditi, L. R. Ginzburg, and H. R. Akcakaya, Variation in plankton densities among lakes: a case for ratio-dependent predation models, Amer. Natur. 138 (1991), 1287-1296.

[3] R. Arditi, N. Perrin, and H. Saiah, Functional response and heterogeneities: an experimental test with cladocerans, OIKOS. 60 (1991), 69-75.

[4] R. Arditi and H. Saiah, Empirical evidence of the role of heterogeneity in ratio-dependent consumption, Ecology 73 (1992), no. 5, 1544-1551.

[5] E. Beretta and Y. Kuang, Global analyses in some delayed ratio-dependent predator-prey systems, Nonlinear Anal. 32 (1998), no. 3, 381-408.

[6] F. D. Chen, Positive periodic solutions of neutral Lotka-Volterra system with feedback control, to appear in Appl. Math. Comput.

[7] F. D. Chen et al., Positive periodic solutions of a class of non-autonomous single species population model with delays and feedback control, to appear in Acta Math. Sinica.

[8] F. D. Chen, F. X. Lin, and X. X. Chen, Sufficient conditions for the existence positive periodic solutions of a class of neutral delay models with feedback control, Appl. Math. Comput. 158 (2004), no. 1, 45-68.

[9] F. D. Chen and S. J. Lin, Periodicity in a logistic type system with several delays, Comput. Math. Appl. 48 (2004), no. 1-2, 35-44.

[10] F. D. Chen, D. Sun, and J. Shi, Periodicity in a food-limited population model with toxicants and state dependent delays, J. Math. Anal. Appl. 288 (2003), no. 1, 136-146.

[11] S. Chen, F. Wang, and T. Young, Positive periodic solution of two-species ratio-dependent predator-prey system with time delay in two-patch environment, Appl. Math. Comput. 150 (2004), no. 3, 737-748.

[12] Y. Chen, New results on positive periodic solutions of a periodic integro-differential competition system, Appl. Math. Comput. 153 (2004), no. 2, 557-565.

[13] J. M. Cushing, Integrodifferential Equations and Delay Models in Population Dynamics, Lecture Notes in Biomathematics, vol. 20, Springer-Verlag, Berlin, 1977.

[14] M. Fan and K. Wang, Periodicity in a delayed ratio-dependent predator-prey system, J. Math. Anal. Appl. 262 (2001), no. 1, 179-190.

[15] M. Fan, Q. Wang, and X. Zou, Dynamics of a non-autonomous ratio-dependent predator-prey system, Proc. Roy. Soc. Edinburgh Sect. A 133 (2003), no. 1, 97-118.

[16] R. E. Gaines and J. L. Mawhin, Coincidence Degree, and Nonlinear Differential Equations, Lecture Notes in Mathematics, vol. 568, Springer-Verlag, Berlin, 1977. 
[17] L. R. Ginzburg and H. R. Akcakaya, Consequences of ratio-dependent predation for steady state properties of ecosystems, Ecology 73 (1992), no. 5, 1536-1543.

[18] A. P. Gutierrez, Physiological basis of ratio-dependent predator-prey theory: the metabolic pool model of Nicholson's blowflies as an example, Ecology 73 (1992), no. 5, 1552-1563.

[19] Z. Jin, S. L. Yuan, and Z. E. Ma, Global existence of positive periodic solutions of a food chain system with Holling type functional response, Gongcheng Shuxue Xuebao 18 (2001), no. 4, 69-75.

[20] B. Li, The existence of positive periodic solution for three-species predator-prey diffusion delay models with functional response, J. Biomath. 17 (2002), no. 4, 385-394.

[21] D. J. Tan, Persistence for a three-species ratio-dependent predator-prey system, J. Biomath. 18 (2003), no. 1, 50-56.

[22] W. Wang and L. Chen, A predator-prey system with stage-structure for predator, Comput. Math. Appl. 33 (1997), no. 8, 83-91.

[23] W. Wang, G. Mulone, F. Salemi, and V. Salone, Permanence and stability of a stage-structured predator-prey model, J. Math. Anal. Appl. 262 (2001), no. 2, 499-528.

[24] D. Xiao and W. Li, Stability and bifurcation in a delayed ratio-dependent predator-prey system, Proc. Edinb. Math. Soc. (2) 46 (2003), no. 1, 205-220.

[25] D. Xiao and S. Ruan, Global dynamics of a ratio-dependent predator-prey system, J. Math. Biol. 43 (2001), no. 3, 268-290.

[26] Y. Xiao, Study on the eco-epidemiology dynamical system, Ph.D. thesis, Academy of Mathematics and System Sciences, Chinese Academy of Sciences, Beijing, 2001.

[27] R. Xu and L. Chen, Persistence and global stability for a three-species ratio-dependent predatorprey system with time delays in two-patch environments, Acta Math. Sci. (English Ed.) 22 (2002), no. 4, 533-541.

[28] R. Xu and L. S. Chen, Persistence and global stability for a three-species ratio-dependent predatorprey system with time delays, J. Systems Sci. Math. Sci. 21 (2001), no. 2, 204-212 (Chinese).

[29] R. Xu, P.-h. Yang, H.-y. Feng, and Z.-q. Wang, Persistence and stability in a delayed ratiodependent ecological system with one predator and two preys, J. Biomath. 17 (2002), no. 2, $149-156$.

Fengde Chen: College of Mathematics and Computer Science, Fuzhou University, Fuzhou, Fujian 350002, China

E-mail address: fdchen@fzu.edu.cn 


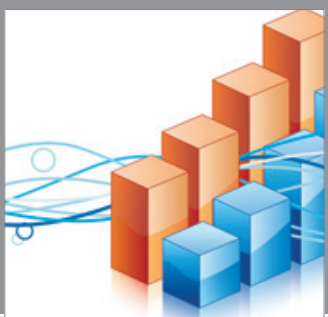

Advances in

Operations Research

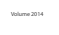

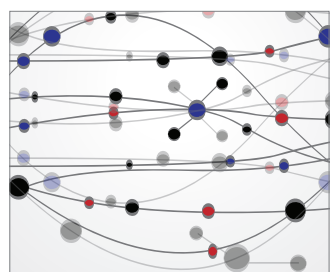

\section{The Scientific} World Journal
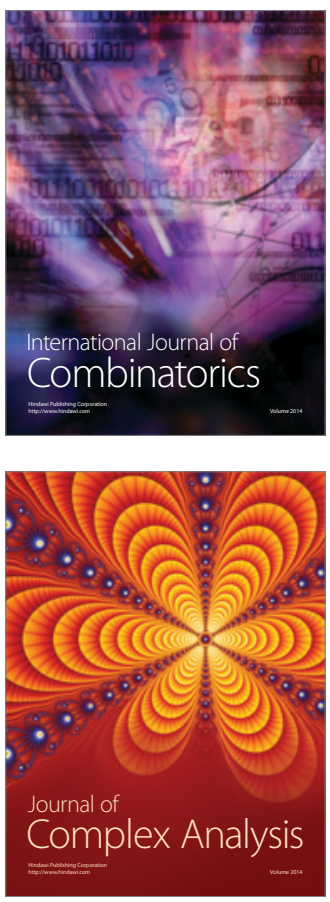

International Journal of

Mathematics and

Mathematical

Sciences
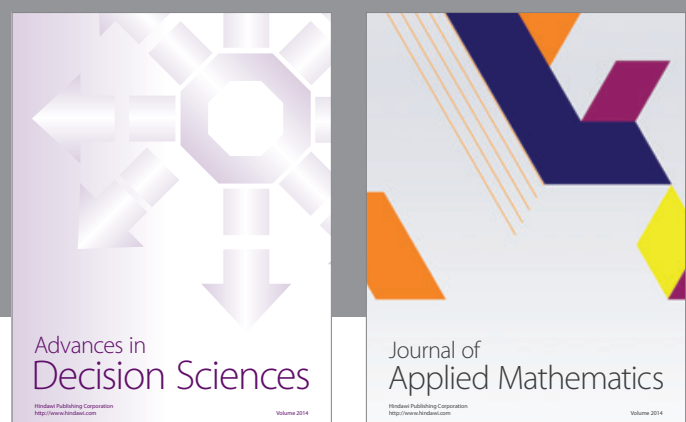

Journal of

Applied Mathematics
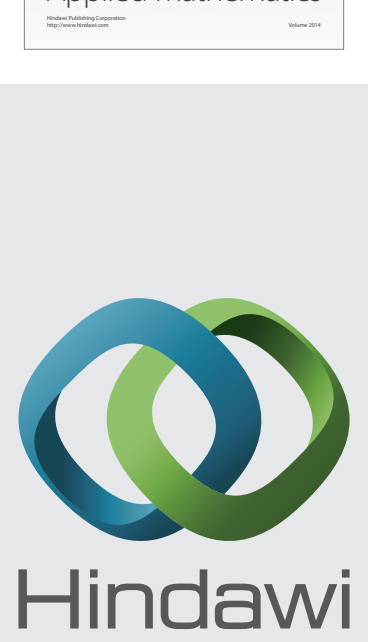

Submit your manuscripts at http://www.hindawi.com
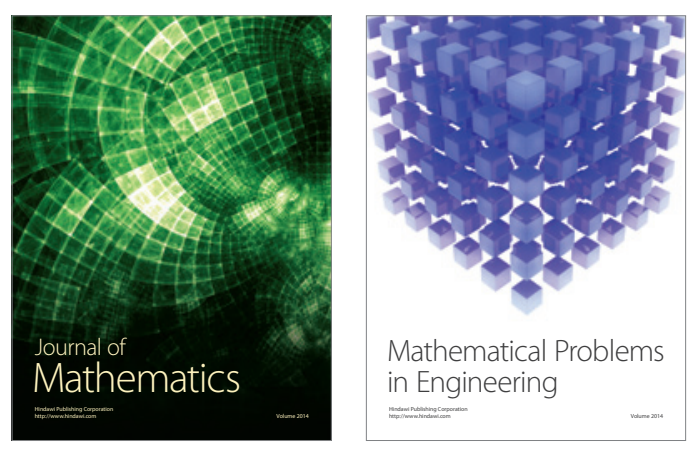

Mathematical Problems in Engineering
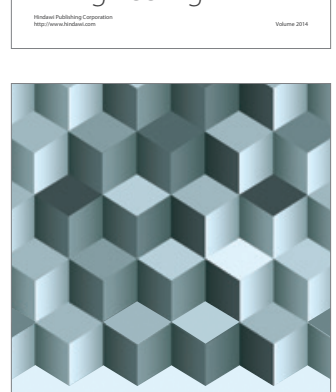

Journal of

Function Spaces
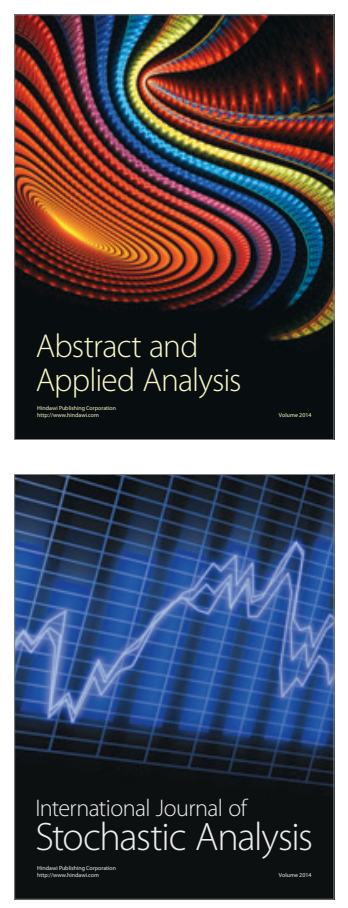

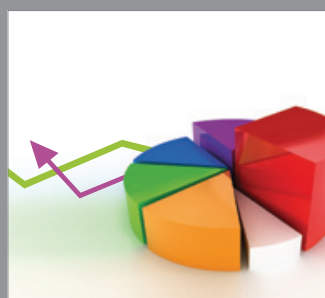

ournal of

Probability and Statistics

Promensencen
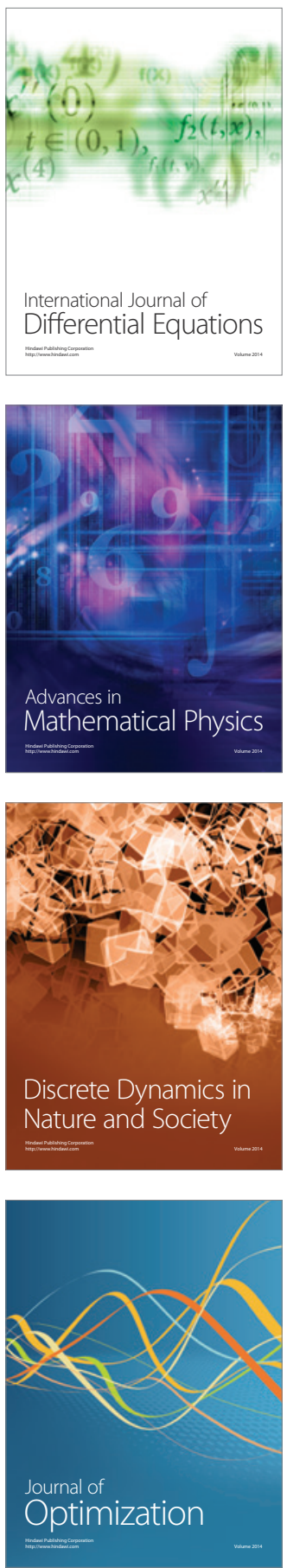